\title{
A Corpus-based Investigation of Actually as a Pragmatic Marker by Chinese Learners of English
}

\author{
Wang Li \\ College of Foreign languages, Nantong University, Nantong, China
}

Copyright $(\mathcal{C} 2015$ by authors, all rights reserved. Authors agree that this article remains permanently open access under the terms of the Creative Commons Attribution License 4.0 International License

\begin{abstract}
This study examines and compares the production of Actually as a pragmatic marker by native and non-native learners of English based on two parallel corpora: BNC (a corpus of spoken British English) and SECCL (a spoken corpus of Chinese EFL learners discourse). The results indicate that actually occurs more frequently in the native speaker corpus than the Chinese learner corpus. The native speakers displayed more varied pragmatic function pattern by employing five pragmatic functions while the Chinese EFL speakers used only one function. Two functions constitute a statistically significant difference between the two speaker groups i.e. the topic shifter and the face-threat function. Nonnative speakers only focus on the face-threatening function.
\end{abstract}

Keywords Pragmatic Marker, Corpus-based, Chinese Learners of English

\section{Introduction}

The functions of the word actually has been analyzed by several previous researchers, and found with high frequency in the English language (Aijmer[1]; Watts[17]). According to Macmillan English Dictionary: For Advanced Learners of American English, there are four different uses of actually: (1) used for emphasizing what is really true or what really happened; (2) used for emphasizing that something is surprising; (3) SPOKEN used when correcting what someone has said or thinks, or what you yourself have said; (4) SPOKEN used for admitting something. While the former two uses serve to emphasize either something true or surprising in written language, the latter two serve to either admit something or express an opinion politely in spoken language. Note that the latter two are more likely to be used in spoken language than in written language.

In the Collins Cobuild Advanced Learner's English Dictionary, the word actually is printed with a three-star rating, showing that it is one of the most basic words in English. This dictionary also categorizes four uses of actually:

1. You use actually to indicate that a situation exists or happened, or to emphasize that it is true.

2. You use actually when you are correcting or contradicting someone.

3. You can use actually when you are politely expressing an opinion that other people might not have expected from you.

4. You use actually to introduce a new topic into a conversation.

Among the above uses, only the first use of actually serves to emphasize something true in written language. As for the others, actually is used to contradict someone, to express an opinion, and to introduce a new topic into a conversation in spoken language. Note that the latter three are more likely used in spoken language than in written language.

In fact, the use of actually has been listed as a pragmatic marker( henceforth PM) by several researchers (Watts[17]). In order to distinguish the role of actually in spoken language from that in written language, Aijmer [1]made use of the London-Lund Corpus of Spoken English and the Lancaster-Oslo Bergen Corpus. She found that actually occurred ten times more frequently in spoken language than in written language. In addition to the analysis of actually in British English, she also compared spoken British English with spoken American English, and pointed out that the use of actually in spoken British English was approximately twice as frequent as that in spoken American English.

To differentiate non-marker use from marker use, Lenk[9] investigated the use of actually in British and North American English. She describes three functions of 'actually' in its discourse marker role: opinion marker, objective/ correction marker, and topic shift marker. She found that actually was used to express pragmatic meanings more often by the British (55.4\%) than the North American (43.94\%).

$\mathrm{Oh}[11]$ analyzes functions of actually in terms of the different utterance positions where actually occurs. Oh proposes that actually serves equally to mark disagreement, contradiction, correction, self-repair, a topic shift or a topic change, whether occurring utterance-initially or utterance-finally. Moreover, $\mathrm{Oh}[11]$ proposes that actually 
functions as a mitigator when it is used to contradict, disagree, or correct a prior view.

Smith and Jucker[14] stress the marker use of actually, by which speakers are able to implicitly express their attitudes towards the propositional content of utterances and show that actually is used to downgrade the force of marking a counterclaim.

Cheng and Warren[5] make efforts to examine the use of actually in intercultural conversations. They study the use of actually among native English speakers and non-native English speakers in Hong Kong, using a corpus of naturally occurring conversations between native and non-native English speakers in Hong Kong. The frequency with which the two groups of speakers use actually and the functions it performs are compared and contrasted. They found that Hong Kong Chinese speakers of English used actually to convey pragmatic meanings far more frequently than did the native English speakers. For instance, the non-native English speakers use actually approximately three times more often than the native English speakers did to mitigate correction or a counterclaim. The patterns of usage are remarkably similar in certain respects but there are differences in use and in the position actually occupies in utterances which in turn can affect the way in which it functions. They claim that actually is used to convey various marker meanings when it appears in peripheral positions, such as the utterance-initial, post-head and utterance-final positions. In general, the functions of actually in such peripheral positions includes mitigating correction, rephrasing, or contradiction, introducing a new topic, a personal point of view or something unexpected, marking friendliness, solidarity or intimacy, and serving as a filler. According to Cheng and Warren[5], the post-head position is between a main clause and a subordinate clause, or between a head and a modifier.

Taglicht[16] attempts to present an overall analysis of actually in phonological, semantic, syntactic, and pragmatic aspects. He distinguishes a "mild" actually from a "strong" actually, and pointes out that the "mild" actually occurs marginally in syntactic structure while the "strong" actually always appears medially in syntactic structure. The "mild" actually functions as either a propositional modifier in pointing to an incompatibility between propositions, or a discourse modifier, which points to a topic change. As regards the "strong" actually, it might be scalar, truth-insistent or WH-insistent. While the "strong" actually exists integrally in syntactic structure and functions as an intensifier, the "mild" actually occurs marginally in syntactic structure and serves as a pragmatic marker.

Clift[6] analyses the pragmatic marker use of actually in terms of turn-constructional units (henceforth TCUs), and proposes three main functions of actually, namely, informing, self-repair and topic movement. Although actually can equally perform the three functions in both TCU-initial and TCU-final positions, these functions are found slightly varied according to the different positions. For the function of informing, a TCU-initial actually can be used to inform the hearer in a soft way that the speaker wants to change his mind or has a different view, whereas a TCU-final actually may be elicited by a question and serves to express the speaker's different view. For the function of self-repair, a TCU-initial actually may be used to mark self-correction due to difficulty in interaction, while a TCU-final actually can be simply introduced as parenthetical self-correction. Finally, for the function of topic movement, a TCU-initial actually, triggered by a prior talk, can function to mark a topic shift, but a TCU-final actually may occur to mark a topic change.

Since the non-marker use of actually, as discussed above, is much less complicated than the marker use of actually in discourse, the following discussion will focus on the latter. To explore the functions of the marker actually, the following discussion will begin with the views of those who did not stress the relation between functions and positions, followed by the views of those who centered on the relation between functions and positions, and the sum of the various functions proposed by previous researchers.

In general, the overwhelming majority of the researchers were in agreement on the distribution that actually serves as a marker in an initial or a final position, and as an adverbial intensifier in a medial position, both in terms of utterance structure and TCU. Nonetheless, Oh[11] proposed that actually could be used either as a marker or an adverbial intensifier in an utterance-medial position. To support his argument, $\mathrm{Oh}[11]$ cited only one example, in which an utterance-medial use of actually was preceded by an utterance-initial use of actually. As for this, more supporting data should be required to examine whether the force of disagreement was triggered by the utterance-medial actually or the utterance-initial actually, since they both can be used to preface forthcoming contradiction.

\section{Research Methodology}

\subsection{Corpora Used in the Present Study}

The present paper uses two corpora for the comparative study: SECCL and BNC. The use of corpora enables a relatively large number of spoken discourses to be examined in empirical analyses of the pragmatic functions of the PMs.

The SECCL corpus (the Spoken English Corpus of Chinese learners) is a sub-corpus of Spoken and Written English Corpus of Chinese learners (SWECCL), which is constructed by Nanjing University in China with a total of one million running words. The corpus is made up of the recordings of 1996-2002 National Spoken English Test for second-year English majors (Band 4). The test consists of three test tasks: Task 1-retelling a story, Task 2- monologue (talking on a given topic) and Task 3- dialogue (role-playing). This research focuses on spoken interactive discourse. Thus, specifically, 105,259 words sample material is chosen from Task 3 for the present study. 


\subsection{Theoretical Framework}

\subsubsection{Non- PM Use of Actually}

When served as a non-marker, the use of actually serves merely to emphasize the truth-value of a proposition in spoken discourse. For instance, in the following discussion about the pros and cons of Hormone replacement therapy (HRT), a TV guest just used actually to emphasize what he found in reality.

(1) TV guest: May I just say, I thi, I think it's important to point out that H R T isn't a miracle cure! And although it will grea, greatly help some women, unfortunately some women are unable to tolerate it, the side effects it gi, it gives them are worse than the actually symptoms they're having in the first place. It means having periods back again which after, and now, er several years break and erm, many women find unacceptable. We all have to have that.

\subsubsection{PM Use of Actually}

In addition to the non-marker use, it is found that actually in my data serves the following pragmatic marker use: 1) to express the speaker's attitudes towards the propositional content; 2) to signal a topic shift or a topic change; 3) to fill the gaps in context while the speaker is still formulating an utterance; 4) to initiate a direct speech report; 5) to preface a face-threatening act. Among these pragmatic functions, the former two fall into the rhetorical structure, and the latter two are classified into the rhetorical structure and the sequential structure respectively.

Regarding the functional analysis, most of the tokens in the corpora served more than one function at the same time. As Andersen[3] pointed out, the functions of actually is not so clear-cut and easily distinguished due to their multi-functionality. When it came to the situation, therefore, only the most dominant function of actually was extracted from its context.

\subsubsection{Actually in the Rhetorical Structure}

The pragmatic functions in the rhetorical structure include: to shift a topic, to make a comment towards the previous proposition and to create time to think.

\section{- Delayer}

(2) TV host: what if they, if they love us back? Oh yes actually hoping so. Yes?

Guest: I think its a bit of a simplification to say that its, its nature's way of keeping us going, because actually er ro, the idea that romantic love is the start of a life long relationship that produces off spring is really quite recent, erm for, for most of history er marriage's were on the basis of continuing er lines, continuing property and people had to erm some how or other cope with living with ano another person that might not necessarily have been the person that they would of chosen from love and, and this is still true in many societies and situations now.

In example (2), they were having a discussion about love.
The guest is obviously using actually to create time to think.

\section{- Evaluator}

(3) TV host: Assuming you should change attitudes, I mean I mean would you agree with Angela that we should change attitudes? Because wha we're talking about a very age- old attitude which is that a mother and a father and their children ar, is the correct unit, should stay together, at least until the children are grown up and probably actually beyond? Alright. I mean, is that an attitude that, that, that should be changed? And if so, how do you do it? Yes?

Guest: Er, I think that actually, the the parents, the mother and father they just don't work hard enough at making a marriage work!

In example (3), they were having a discussion about family issues. The TV host inquires about whether the attitude should be changed and the guest responded by using I think and actually to express his opinion towards the host's question. This use of actually fits in with what Lenk[9] proposed when actually is used as an opinion marker, it is frequently associated with the phrase I think.

\section{- Topic shifter}

According to Lenk [9], actually may function as a topic shift marker in discourse. And, as a topic shift marker, it often occurs together with you know.

(4) Guest: oh, it was feel, it was so terrifying, you just, you regard every one with suspicion after that.

TV host: $\mathrm{mm}, \mathrm{mm}$

Guest: I actually feel, there was a time I used to think it wouldn't happen to me because if I was in that situation I would do this, I would do the next thing and when it did actually happen to me, it took me ages to get over

In example (4), the use of actually serves to mark a topic shift. This dialogue took place between a TV host and a guest. They were having a discussion about crime.

\subsubsection{Actually in the Sequential Structure}

\section{Direct speech initiator}

(5) TV host: I should ask you actually which is a, have you ever been in an accident? erm hang on a minute, button one for yes, button two for no, have you ever been in an accident? Now you see this is an extraordinary figure, you all...

In this dialogue, $\mathrm{TV}$ host is investigating how many people have ever been in an accident. He uses actually to elicit his question.

\subsubsection{Actually in the Inferential Structure}

There is only one pragmatic function employed in the inferential structure in my data: face-threat mitigator.

\section{1) Face-threat mitigator}

This dialogue took place when two students are discussing whether the Geology department should stick to its original plan to enroll more male students.

(6) A: But I think, you know, according to some rules and 
to some... eh...... statistic in some riss newspapers. we should live up to this standards and I think this priportion is ri reasonable. Because there are still 5 females can... admit to this university.

B: Eh... m, actually I I I can't with agree with you.

A: Eh...?

B: Eh... $\mathrm{m}, \mathrm{I}$ think it is a a discrimination. I think.

In example (6), A and B are discussing whether the Geology Department should stick to its original plan to enroll more male students. B uses actually to mitigate his tone to object to A's opinion.

\section{Results and Discussion}

\subsection{The Use of Actually in BNC and SECCL}

According to Table 1, there are considerable differences in the use of actually between the two groups of speakers. In terms of frequency, the total token frequency of actually among the native speakers is much higher than that of actually employed by non-nonnative speakers. Actually ranks the $86^{\text {th }}$ in BNC wordlist, which means it is the $86^{\text {th }}$ frequently used word among native speakers while it is only the $470^{\text {th }}$ frequently used word among non-native speakers. However, as regards pragmatic functions, there are eleven pragmatic uses of actually out of a total of 14 occurrences of actually (78.6\%). The PM use of actually is used roughly seven times by non-native speakers more than native speakers do $(78.6 \%$ vs. $11.4 \%)$. In fact, such a finding may accord with Cheng and Warren's[5] research result that in Hong Kong non-native English speakers use pragmatic actually much more often than native English speakers do. They found that non-native English speakers use pragmatic actually three times more often than native speakers do. In this case, it is assumed that native Chinese speakers may be influenced by their first language so that they use actually frequently and even improperly in some situations when they speak English.

Table 1. Frequency of actually in BNC and SECCL

\begin{tabular}{|c|c|c|}
\hline & BNC & SECCL \\
\hline Total number of words & 105,337 & 105,259 \\
\hline Total number of actually & 202 & 14 \\
\hline Number of PM & 23 & 11 \\
\hline P-function ratio of actually (\%) & $11.4 \%$ & $78.6 \%$ \\
\hline Rate of PM actually (per 10000 words) & 2.3 & 1.1 \\
\hline Total Rank in Wordlist & 86 & 470 \\
\hline
\end{tabular}

In the following, functional differences among the three pragmatic structures between the two groups will be showed in Table 2.
Table 2. Contrast of overall pragmatic functions of actually in BNC and SECCL

\begin{tabular}{|c|c|c|c|c|}
\hline $\begin{array}{c}\text { Coherence } \\
\text { relations }\end{array}$ & Functions & BNC & SECCL & Z-score \\
\hline \multirow{2}{*}{ Rhetorical } & DEL & $3(13 \%)$ & $0(0 \%)$ & +1.25 \\
\cline { 2 - 5 } & EVA & $4(17.4 \%)$ & $0(0 \%)$ & +1.47 \\
\cline { 2 - 5 } & TOP & $10(43.5 \%)$ & $0(0 \%)$ & +2.6 \\
\hline Sequential & DIR & $1(4.3 \%)$ & $0(0 \%)$ & +0.7 \\
\hline Inferential & FAC & $5(21.8 \%)$ & $11(100 \%)$ & -4.28 \\
\hline Total & N\% & $23(100 \%)$ & $11(100 \%)$ & \\
\hline
\end{tabular}

Table 2 presents the frequency distribution within each pragmatic function of actually in the two spoken corpora. As a PM, actually was observed to function in five ways in the BNC corpus whereas in SECCL only one function of actually was found (i.e. Face-threat mitigator function). In the case of individual functions, the non-native speakers tend to significantly under-use the function as topic shifter $(p<0.01)$ and significantly overuse the function as fact-threat mitigator $(p<0.01)$. Native speakers, by contrast, employed nearly half of the whole pragmatic use of actually $(43.5 \%)$ for the topic-shifting purpose. Comparatively speaking, actually is more evenly distributed among all the functions in BNC while non-native speakers seems to have a great preference for face-threat mitigator over the other functions in SECCL. The rest of the pragmatic functions are not found in the non-native corpu. This study is accordance with Cheng and Warren[5]'s analysis of the pragmatic functions of actually in the Hong Kong corpus of spoken English. They found that actually was used three times more often by L2 speakers pragmatically than their native counterparts. While native speakers groups used it to emphasize that something unexpected is in fact correct, to mitigate a correction, rephrasing or contradiction, and to introduce a new topic, L2 speakers used it more often to act as a face-threat mitigator. The motive behind the frequent use of PMs as face-threat mitigator function can be attributed to dissimilar face systems and notions of politeness of the Chinese (Gu [7]), compared to those of the West, hence contributed to differing purposes of language use in communication.

The notion of "face" of an individual is derived from that of $\mathrm{Hu}[8]$ which links face with notions of being embarrassed or humiliated, or "losing" face. Thus face is something that is emotionally invested and that can be lost, maintained or enhanced, and must be constantly attended to in interaction. Based on Goffman's notion of "face", Brown and Levinson [4] propose that face as a public self-image is composed of two specific face-wants: positive and negative face. "Positive face" refers to the positive consistent self-image or "personality" (crucially including the desire that this self-image be appreciated and approved of) claimed by interactants. "Negative face" refers to an individual's basic claim to territories, personal preserves, rights to non-distraction, i. e. to freedom of action and freedom from imposition (Brown and Levinson [4]). 
Table 3. Distribution of actually in respective pragmatic structure ( $\mathrm{N}$ and \%).

\begin{tabular}{|c|cc|cc|c|}
\hline \multirow{2}{*}{ Category } & \multicolumn{2}{|c|}{ BNC } & \multicolumn{2}{c|}{ SECCL } & \multirow{2}{*}{ Z-score } \\
\cline { 2 - 5 } & Frequency & Proportion & Frequency & Proportion & \multirow{2}{*}{$+4.03 *$} \\
\hline Rhetorical & 17 & $73.9 \%$ & 0 & $0 \%$ & +0.70 \\
\hline Sequential & 1 & $4.3 \%$ & 0 & $0 \%$ & $-4.28 \cdots$ \\
\hline Inferential & 5 & $21.8 \%$ & 11 & $100 \%$ & \\
\hline Total & 23 & $100 \%$ & 11 & $100 \%$ & \\
\hline
\end{tabular}

Face systems and politeness values in Chinese are different from those in western cultures . Chinese "face" has two aspects: "mianzi" which refers to one's need to conform to social conventions and to express one's desire to be part of the community $(\mathrm{Hu}[8])$, and "lian" which defines one's need to show a moral sense of place and role. Both roles revolve around an individual's desire for others to recognize his or her social prestige, reputation or sanction $(\mathrm{Hu}[8])$. In the West, egalitarianism in relationships is a valued cultural ideology; while in Asia, hierarchical human relationships are valued. Westerners and Asians hence differ in the way they conceive face. Westerners emphasize the independence aspect of face. They respect the rights of the addressee to freedom of choice, autonomy, territory, and so on. For Asians, due to the valued hierarchical human relationships as a cultural ideology, they tend to emphasize the involvement aspect of face; and that is, the importance of "being a normal and contributing participant in communicative events" (Scollon and Scollon[13]).

Chinese politeness centres on self-denigration and other elevation $(\mathrm{Gu}[7])$. The Chinese word for "politeness" is "limao", the word-for-word translation of which is "polite appearance" $(\mathrm{Gu}[7])$. "limao" is formulated by Confucius (551 B. C. - - 479 B. C.) and is derived from the Chinese word "li", which refers to "the social hierarchy and order of the slavery system of the Zhou Dynasty (dating back to $1100 \mathrm{~B}$. C.)". "Li" itself conceals two meanings: social hierarchy and order and politeness, which are closely connected to each other. Social hierarchy and order give rise to politeness, and politeness expresses and helps to maintain social hierarchy and order $(\mathrm{Gu}[7])$.

As proclaimed by Markus and Kitayama [10], Asian cultures support the "interdependent conception of the self" more than Western cultures. An "interdependent Self" is defined by the person's relations with others in specific contexts (Markus and Kitayama[10]). The interdependent construal of self is more likely to pay attention to the group when forming opinions and attitudes, to attach importance to preserving their own and others" "face", and emphasize harmony and cooperation in the in group. The other-orientation is therefore essential to an interdependent self. Congruous with the notion of an interdependent self, the Chinese self, in the Confucian sense, is defined by a person's surrounding relations.

Table 3 presents the pragmatic functions within each pragmatic structure. Among all the pragmatic functions employed by native speakers, the rhetorical structure outnumbered significantly the others in the sequential structure $(p<0.01)$. However, Chinese EFL learners, by contrast, overuse significantly the functions in the inferential structure $(p<0.01)$. They tend to focus only on the face-threatening function. A possible explanation for its dominant presence in the inferential structure is mainly due on the one hand to a non-L2 like pragmatic knowledge stemming from a lack of appropriate input and on the other hand to deficiencies in pragmatic ability explained by insufficient opportunities of use.

\subsection{Comparison of Positions of Actually between BNC and SECCL}

In BNC and SECCL, two positions of you know have been observed. Table 4 illustrates the comparative frequencies in position placement between $\mathrm{BNC}$ and SECCL.

Table 4. Comparison of positions of actually between BNC and SECCL

\begin{tabular}{lccccc}
\hline $\begin{array}{c}\text { Coherence } \\
\text { relations }\end{array}$ & & \multicolumn{2}{c}{ BNC } & \multicolumn{2}{c}{ SECCL } \\
\hline \multirow{4}{*}{ Rhetorical } & Functions & Initial & Middle & Initial & Middle \\
\cline { 2 - 6 } & DEL & $0(0 \%)$ & $3(100 \%)$ & $0(0 \%)$ & $0(0 \%)$ \\
\cline { 2 - 6 } & EVA & $0(0 \%)$ & $4(100 \%)$ & $0(0 \%)$ & $0(0 \%)$ \\
\cline { 2 - 6 } TOP & $0(0 \%)$ & $10(100 \%)$ & $0(0 \%)$ & $0(0 \%)$ \\
\hline Sequential & DIR & $0(0 \%)$ & $1(100 \%)$ & $0(0 \%)$ & $0(0 \%)$ \\
\hline Inferential & FAC & $0(0 \%)$ & $5(100 \%)$ & $11(100 \%)$ & $0(0 \%)$ \\
\hline Total & No & $0(0 \%)$ & $23(100 \%)$ & $11(100 \%)$ & $0(0 \%)$ \\
\hline
\end{tabular}

Results of this study reveal broad diversities in the function of actually compared with other analyses of researchers. Analysis of distribution in BNC (see Table 4) shows that all the cases of pragmatic actually in the native corpus $(100 \%)$ were medial; initial and final positions were absent from the present study. This is notably different from frequencies reported by Aijmer[1] in the London-Lund corpus of spoken English, where only $32 \%$ of occurrences were medial and Oh [11] who found $34 \%$ of all uses of actually were initial, $58 \%$ were medial and $8 \%$ were final in the corpus of spoken American English. In both the initial and the final position, the primary function of actually was to introduce something in the discourse that was unexpected in some way. In this use, it gently contradicted, corrected or 
disagreed with the expectation set up by the prior utterance. The medial position typically functioned as an emphasizer, reinforcing and strengthening the truth-value of the clause. In contrast, analysis of distribution of actually in SECCL revealed that Chinese EFL learners tend to place all of the occurrences of actually in the initial position.

The present study also disagrees with Oh's[11]assertion that actually is highly unlikely to co-occur with the verbs think, feel or believe, because of the possibility of some ambiguity in its function between emphatic strengthening or gentle contradiction. According to him, these verbs prefer to pattern with really to indicate emphasis unambiguously, and avoid any suggestion that disagreement is being signaled $(\mathrm{Oh}[11])$. This finding was not strongly supported in both the native corpus and non-native corpus, which showed high frequencies of collocation with verbs of think, feel or believe.

\section{Conclusions and Implications}

All in all, actually occurs more frequently in the native speaker corpus than the Chinese learner corpus. The native speakers displayed more varied pragmatic function pattern by employing five pragmatic functions in my data while the Chinese EFL speakers used only one function. Two functions constitute a statistically significant difference between the two speaker groups i.e. the topic shifter and the face-threat function. They only focus on the face-threatening function.

Since the use of actually can contribute to naturalness and interactivity of speech, this deficiency in the use of actually results in an unnatural and awkward speech patterns. According to Wilson and Sperber [15], pragmatic markers encode procedural meaning and cannot be brought to consciousness. According to Schmidt[12], only when input is noticed can it become intake and effective processing. Therefore, a degree of awareness is important before input can be incorporated into a developing interlanguage system. Theoretically speaking, the more frequent the use of actually as pragmatic markers is in the input stream, the more likely it is to be noticed and then become integrated into the interlanguage system. Thus, it is suggested by the author that, in order to facilitate their acquisition of actually as pragmatic markers, the learners must be exposed more to spoken discourse and be instructed on the use of actually. Subsequently, the more actually as pragmatic markers stands out in the input stream, the more likely that it will be noticed. According to Sperber and Wilson [15], pragmatic markers express procedural information and cannot be brought to consciousness. In other words, pragmatic markers are not perceptively salient because they do not express conceptual meaning. Therefore, there is slim chance for actually as pragmatic markers to be noticed and acquired by the learners.

Although the use of actually as pragmatic markers are not perceptively salient, the learners' awareness can be aroused by way of instruction. Instruction can make relevant the less obvious aspects of the input. It channels attention and brings into awareness what otherwise would have been missed. Considering that pragmatic markers are not easily noticed, instruction seems quite necessary in the case of its pragmatic marker acquisition. Otherwise, they would often be neglected. What the present oral discourse falls short of is the opportunity to focus on its use as pragmatic markers. So, it is advocated here that formal instruction on pragmatic marker use be introduced.

Only when this is done can acquisition of actually as pragmatic markers be more efficient and fruitful. Processing ability concerns the learner's capacity to deal with the range of forms in input. A person's processing ability is limited. When part of the capacity is occupied with one task, less is available for another. So when the learners concentrate more on meaning or forms rather than pragmatic markers, they are less likely to notice the use of actually as pragmatic markers, and thus the acquisition is less likely to take place.

In order to make the learners fully aware of the use of actually as pragmatic markers, tasks should not be too demanding. Other aspects of language should not distract them too much to make sure that enough capacity is available for the processing of pragmatic markers. According to two studies on the acquisition of pragmatic markers by young children, the acquisition of pragmatic markers tends to develop from that of the literal meanings to that of the pragmatic meanings (Andersen[3]). For the learners who have acquired the literal meanings of pragmatic markers, a prediction can be made that noticing and acquiring the pragmatic functions of pragmatic markers will subsequently take place, because it is the "next" thing to be acquired. Finally, according to Schmidt [12], more demanding tasks consume more attention resources for task transaction, thereby leaving less attention available for focus on form. When a learner's limited processing capacity system is overloaded, noticing is less likely to take place. So the design of tasks for formal instruction on pragmatic markers should take task-difficulty into consideration.

In conclusion, the lack of frequent input as well as perceptive salience and instruction may lead to the learners' failure to acquire the use of actually as pragmatic markers. To ensure their acquisition of actually as pragmatic markers, formal instruction should be adopted so that its pragmatic marker use are frequent and prominent enough to be noticed then acquired. Formal instruction should take processing capacity and the current state of the learners' interlanguage into consideration, as well task difficulty.

\section{Acknowledgements}

The paper derived from a research project, entitled "An investigation into the acquisition of Chinese discourse markers by learners from different language backgrounds" (13YJC740096), sponsored by the Educational Council of the Chinese government in the 2013-2016 academic year. 


\section{REFERENCES}

[1] Aijmer, K. (2002). English Discourse Particle. Evidence from a Corpus. Amsterdam: John Benjamins.

[2] Andersen, G. (Ed.) (1998). The pragmatic marker like from a relevance-theoretic perspective. In A. Jucker, \& Y. Ziv (Eds.), Discourse markers: Descriptions and theory (pp. 147-170). Amsterdam: John Benjamins.

[3] Andersen, G. (2001). Pragmatic markers and sociolinguistic variation: A relevance-theoretic approach to the language of adolescents. Amsterdam and Philadelphia: John Benjamins.

[4] Brown G, Levinson S. (1978). Some Universals of Language Usage: Politeness Phenomena. London: Cambridge University Press.

[5] Cheng, W. and Warren M.. (2001). The functions of actually in a corpus of intercultural conversations. International Journal of Corpus Linguistics, 6(2),257-280.

[6] Clift, R. (2001). Meaning in interaction: The case of actually. Language, 77(2), 245-291.

[7] Gu, Yueguo. (1990).Politeness phenomena in modern Chinese. Journal of Pragmatics 14: 237-257.

[8] Hu, Hsien Chin.(1944).The Chinese concept of 'face'. American Anthropologist, 46.

[9] Lenk Watts, Richard J. (1988). A relevance-theoretic approach to commentary pragmatic markers: The case of actually, really and basically. Acta Linguistica Hungarica, 38(1-4), 235-260.

[10] Markus, Hazel R. and Kitayama, Shinobu. (1991). Culture and the self: Implications for cognition, emotion, and motivation. Psychological Review, 98: 224-253.

[11] Oh, Sun-Young. (2000). Actually and in fact in American English: A data-based analysis. English Language and Linguistics, 4(2), 243-268.

[12] Schmidt, R. (1990). The role of consciousness in second language learning. Applied Linguistics, 11(2), 120-158.

[13] Scollon, Ron. (1995). Intercultural Communication: A Discourse Approach. Oxford: Blackwell.

[14] Smith, S. W., \& Jucker, A. H. (2000). Actually and other markers of an apparent discrepancy between propositional attitudes of conversational partners. In G. Andersen, \& T. Fretheim (Eds.), Pragmatics markers and propositional attitude (pp. 207-237). Amsterdam and Philadelphia: John Benjamins.

[15] Sperber, D., \& Wilson, D. (1995). Relevance: Communication and cognition. Oxford: Blackwell. (Original work published 1986)

[16] Taglicht, J. (2001). Actually, there's more to it than meets the eye. English Language and Linguistics, 5(1), 1-16.

[17] Watts, R. (1989). Taking the pitcher to the "well": Native speakers' perception of their use of discourse markers in conversation. Journal of Pragmatics, 13. 